\title{
Evaluating landscape complexity and the contribution of non-locality to geomorphometry
}

\author{
Christopher J. Keylock ${ }^{1}$, Arvind Singh ${ }^{2}$, Paola Passalacqua ${ }^{3}$, and \\ Efi Foufoula-Georgiou ${ }^{4}$
}

${ }^{1}$ School of Architecture, Building and Civil Engineering, Loughborough University, Leicestershire, LE11 3TU, UK.

${ }^{2}$ Civil, Environmental and Construction Engineering, College of Engineering and Computer Science, University of Central Florida, FL, USA

${ }^{3}$ Civil, Architectural and Environmental Engineering, Cockrell School of Engineering, University of Texas at Austin, TX, USA ${ }^{4}$ Departments of Civil and Environmental Engineering and Earth System Science, Henry Samueli School of Engineering, University of California Irvine, CA, USA

March 25, 2021

\begin{abstract}
A long-standing question in geomorphology concerns the extent that statistical models of terrain elevations have adequate characteristics with respect to the known scaling properties of landscapes. In previous work, it has been challenging to ascribe statistical significance to metrics adopted to measure landscape properties. In this paper, we use a recently developed surrogate data algorithm to generate synthetic surfaces with identical elevation values to the source dataset, while also preserving the value of the Hölder exponent at any point (the underpinning characteristic of a multifractal surface). Our primary source data are from a laboratory experiment on landscape evolution. This allows us to examine how the statistical properties of the surfaces evolve through time and the extent to which they depart from the simple (multi)fractal formalisms. We show that there is a strong departure that is driven by the diffusive processes in operation. The number of sub-basins of a given channel order (for orders sufficiently small relative to the basin order) exhibit a clear increase in complexity after a steady-state for sediment flux is established. We also study elevation data from Florida and Washington State where the relative departure from simple multifractality is even more strongly expressed but is similar for two very different locations. Our results show that at
\end{abstract}


the very least, the minimum complexity for a stochastic model for terrain statistics with appropriate geomorphic scalings needs to incorporate a conditioning between the pointwise Hölder exponents and elevation.

\begin{abstract}
A long-standing question in geomorphology concerns the extent that statistical models of terrain elevations have adequate characteristics with respect to the known scaling properties of landscapes. In previous work, it has been challenging to ascribe statistical significance to metrics adopted to measure landscape properties. In this paper, we use a recently developed surrogate data algorithm to generate synthetic surfaces with identical elevation values to the source dataset, while also preserving the value of the Hölder exponent at any point (the underpinning characteristic of a multifractal surface). Our primary source data are from a laboratory experiment on landscape evolution. This allows us to examine how the statistical properties of the surfaces evolve through time and the extent to which they depart from the simple (multi)fractal formalisms. We show that there is a strong departure that is driven by the diffusive processes in operation. The number of sub-basins of a given channel order (for orders sufficiently small relative to the basin order) exhibit a clear increase in complexity after a steady-state for sediment flux is established. We also study elevation data from Florida and Washington State where the relative departure from simple multifractality is even more strongly expressed but is similar for two very different locations. Our results show that at the very least, the minimum complexity for a stochastic model for terrain statistics with appropriate geomorphic scalings needs to incorporate a conditioning between the pointwise Hölder exponents and elevation.
\end{abstract}

\title{
1 Introduction
}

A landscape is an assemblage of individual, identifiable features that can be classified and explained by the geomorphologist (Arrell et al., 2007; Ehsani and Quiel, 2008; Passalacqua et al., 2010; Clubb et al., 2014), and also the parts in between that are a palimpsest of current and past processes (Jerolmack and Paola, 2010). A focus on individual landscape features can be key to unlocking the geological history of a region (Kirby and Whipple, 2012; Gasparini et al., 2014), while consideration of the landscape as a whole using measures such as the hypsometric integral (Strahler, 1952; Boon III and Byrne, 1981; Brocklehurst and Whipple, 2004; Keylock et al., 2020b), or statistical scaling laws of topography (Hack, 1957; Tokunaga, 1978; Willgoose, 1994; Lague and Davy, 2003; Zanardo et al., 2013) gives an insight in to how uplift, erosion and deposition interact to shape our landscapes. From the perspective of this latter approach, the question remains as to the extent that statistical models for topography can adequately represent observed elevation statistics. Expressed another way, do the particular dynamics of geomorphic processes leave an imprint on the terrain that makes simple statistical models inadequate? Addressing this question forms the goal of this paper. 
The complex configuration and environmental history of a landscape make a formal, mathematical or statistical description of terrain regularity problematic. Attempts to do this have commonly adopted methods based on the notion of fractal dimension (Klinkenberg and Goodchild, 1992; Lifton and Chase, 1992; Outcalt and Melton, 1992; Gagnon et al., 2006). However, the generalization of the description of landscape from mono-fractal to one where more than a single fractal dimension is present has resulted in significant terminological confusion. Monofractality assumes that the Hölder exponent describing the terrain is constant everywhere (it is a Hurst exponent), while in this study we use the term "multi-Hölder" for the general notion of a terrain where the Hölder exponents vary. This provides the freedom to contrast a "multifractal" surface with a "multi-fractional Brownian surface" in terms of how the variability is structured: A multifractal surface is one that is the outcome of a hierarchical process such that individual Hölder exponents are imbricated in a non-continuous fashion (Benzi et al., 1993), while a multi-fractional Brownian surface is one where the variability is given by a continuous function (Peltier and Lévy Véhel, 1995). In addition, a landscape may be considered as being driven by a self-regulating process, a relatively recent development in regularity theory where the regularity is coupled to the values of the signal (Echelard et al., 2015). This concept underpins the velocity-intermittency method for extracting information on flow structures from turbulence time series (Keylock et al., 2012; Ali et al., 2019; Keylock et al., 2020a), and in the case of landscape geomorphology, would mean that the elevation drives the regularity. This notion of self-regulation underpinned the recent proposal to couple hypsometric (area-elevation analysis) (Strahler, 1952) to Hölder analysis (Keylock et al., 2020b), which is a technique utilized in this study.

While previous work has shown that geomorphic surfaces are not monofractal (Evans and McLean, 1995; Perron et al., 2008), the nature and extent to which a multi-Hölder description of landscape adequately represents geomorphic process is still unclear. While some authors have suggested that a multifractal model is suitable for characterizing topography (Gagnon et al., 2006), others have been more cautious. Of particular importance in this context is a study by Veneziano and Iacobellis (1999) that not only critiqued the methodologies adopted in some previous works, but also showed that for various terrains, there was evidence of consistent self-similar relations for both the channel network part of the terrain where fluvial incision was dominant, and the hillslopes dominated by diffusive processes. In other words, the Hölder exponents in a land surface are conditioned on the type of process that is dominant (an advective fluvial system or a diffusive hillslope one). See Clubb et al. (2020) for recent work that has arrived at a similar conclusion.

A difficulty with all previous investigations of this phenomenon is the absence of an appropriate control that may be used to compare extracted statistical quantities from topographic surfaces and determine their statistical significance. Given that some of these phenomena are rather subtle in nature, as well as the error in any statistical curve-fitting exercise used for deriving a scaling relation, this is important. In this study, we develop a framework that permits analysis 
of landscape scaling properties relative to appropriate control models for the topography. We apply this framework to investigate the extent to which elevation statistics contain a signature from geomorphic processes that cannot be represented adequately by simple multi-Hölder models for terrain statistics. To accomplish this, we make use of a laboratory experiment on terrain evolution (Singh et al., 2015), as well as digital elevation models (DEMs) from Florida and Washington State. Before presenting our methodology and results, we briefly review the definition of the pointwise Hölder exponents that underpin fractal and multifractal analyses.

\section{Hölder exponents}

Given a DEM containing elevations, $z(x, y)$, the increments (the elevation differences between points at separation, $r$ ) are:

$$
\delta z=z(x, y)-z(x+r \cos \theta, y+r \sin \theta)
$$

where $\theta$ is a direction selected for analysis and $r$ is the separation distance between points. The statistical moments of order $n$ for $\delta z$ are given by $\left\langle\delta z^{n}\right\rangle$, where the angled braces indicate an averaging operation. The scaling relation

$$
\left\langle|\delta z|^{n}\right\rangle \propto r^{\xi_{n}}
$$

is then found from a log-log plot of $\left\langle|\delta z|^{n}\right\rangle$ against $r$. A fractal form for the distribution of elevations implies that $\xi_{n}$ increases linearly with $n$ (Frisch and Parisi, 1985). In the well-known case of classical turbulence theory, the Kolmogorov (1941) theory gives $\xi_{n}=\frac{1}{3} n$.

A multi-Hölder signal exhibits a convex relation between $n$, and $\xi_{n}$ (Frisch and Parisi, 1985), but is more formally concerned with the set of pointwise Hölder scaling exponents, $h(x, y)$, that characterize the properties of the surface at all positions, $(x, y)$. At a particular position, $(x=X, y=Y)$ we can evaluate the local scaling behavior of $z$ to determine the Hölder exponent, $h$, in a fashion that is similar to the statistical moments of the increments, above, but without the averaging operator:

$$
|z(X, Y)-z(X+r \cos \theta, Y+r \sin \theta)| \sim C|r|^{h(X, Y)}
$$

where $C$ is a constant (see Venugopal et al. (2006) for a review). Having applied (3) to the whole DEM, (i.e. we have $z(x, y)$ and $h(x, y)$ ), the singularity spectrum, $D(h)$, may be derived as the Hausdorf (fractal) dimension, $d_{H}$, of the set of all points, $(x, y)$, where the $h(x, y)=h$. I.e. $D(h)=d_{H}(x, y)$ belongs to the support of $z(x, y)$ for $h(x, y)=h$. The Frisch-Parisi conjecture states that

$$
D(h)=\min _{n}\left(h n-\xi_{n}+1\right) .
$$

Thus, the structure functions and the Hölder exponents are related via a Legendre transform (Jaffard, 1997). Therefore, a mono-fractal landscape has a 
constant degree of proportionality between $n$ and $\xi_{n}$, giving a single constant value for $D(h)$ : the Hurst exponent, $d$. The fractal dimension of the surface would then be $\mathscr{D}=2+(1-d)$. Given a departure from mono-fractality, there will be multiple values for the Hölder exponents observed in the terrain. These may be organized in a hierarchical fashion (like a true multifractal process) or potentially a function of another variable, such as elevation itself (a self-regulating process). Large Hölder exponents correspond to smoother regions in the terrain and, thus, are potentially related to more diffusive processes, while small exponents are likely linked to incision and advective processes. This geomorphic interpretation motivated Keylock et al. (2020b) to re-examine the area-altitude scaling of terrain (hypsometry) by coupling it to the Hölder exponents, thereby linking topographic roughness to the broader manner in which elevations are distributed in the terrain, leading to an ability to discriminate between landscapes from different areas or with different underlying process-based generating mechanisms, even if all have similar hypsometric integrals.

\section{Methodology}

\subsection{Experimental set-up}

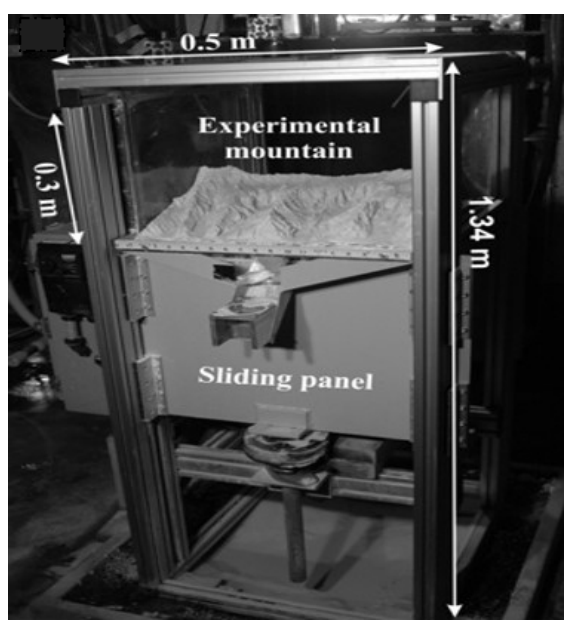

Figure 1: Illustration of the eXperimental Landscape Evolution (XLE) facility at the University of Minnesota.

Experiments were performed at the eXperimental Landscape Evolution (XLE) facility of the St. Anthony Falls Laboratory at the University of Minnesota (Fig. 1). XLE consisted of a $0.5 \times 0.5 \times 0.3 \mathrm{~m}^{3}$ erosion box with two opposing sides able to slide up and down at variable rates mimicking changes in the base level. The facility includes a rainfall simulator consisting of 20 ultrafine misting nozzles which were able to generate rain droplets of sizes less than $10 \mu \mathrm{m}$. The 
experimental setup was also equipped with a laser scanner able to scan the experimental topography at resolution of $0.5 \mathrm{~mm}$ in a few seconds. This was done every 300 seconds for over nine hours. The experimental landscapes discussed here were evolved under constant uplift, $U=20 \mathrm{~mm} \mathrm{~h}^{-1}$, and precipitation intensity, $P=45 \mathrm{~mm} \mathrm{~h}^{-1}$. The erodible material was a homogeneous mixture of fine silica (specific density 2.65) with a grain size distribution of $D_{25}=10 \mu \mathrm{m}$, $D_{50}=25 \mu \mathrm{m}$, and $D_{75}=45 \mu \mathrm{m}$, mixed with $35 \%$ water by volume in a cement mixer; see Singh et al. (2015); Tejedor et al. (2017) for more details. The key changes that arose in the evolution of the topography were the establishment of a drainage basin at $t \sim 150$ minutes, the main drainage divide at $t \sim 225$ minutes, a steady-state landscape in terms of sediment flux at $t \sim 375$ minutes, and a final evolution towards a morphometric steady-state for $t \gtrsim 475$ minutes. Each of these stages is illustrated in Fig. 2. Sediment flux steady state was considered to be achieved when the mean erosion rate did not change with time, with mean erosion rate computed from the differenced DEMs (Singh et al., 2015). In the morphometric steady state, there is no change in the topographic features with time. While the landscape surfaces we generated were primarily erosional, differencing consecutive DEMs did indicate that significant deposition events did arise. These were more prevalent once flux steady state was established as can be seen in Fig. 6 of Singh et al. (2015).
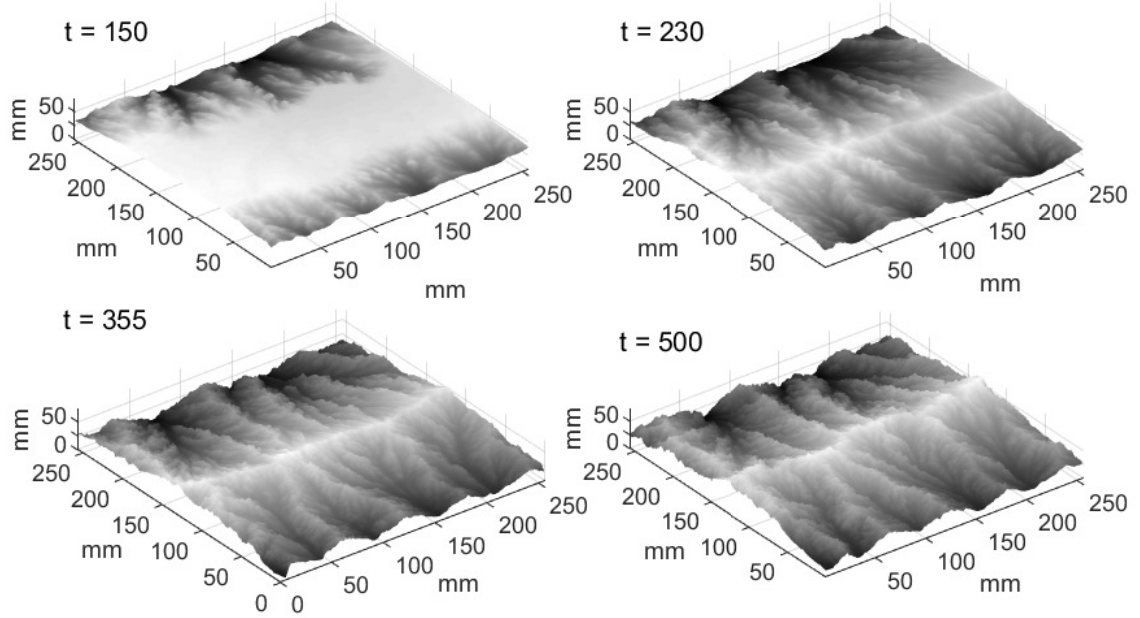

Figure 2: Stages in the evolution of the experimental topography as described in the text. The time intervals quoted are in minutes. 


\subsection{Hypothesis testing with surrogate data}

In order to distinguish between the various forms of regularity that might arise in terrain statistics, we need a suitable means to place statistical confidence on the results. In this paper we use the concept of surrogate data, which have been used for about thirty years for hypothesis testing for non-linear processes in time-series signal processing (Theiler et al., 1992). This field, including geophysical applications of the salient methods, was recently reviewed by Keylock (2019). Example DEMs generated using the most well-known surrogate data generating algorithm, which preserves the Fourier amplitudes of the original data, are provided in the Appendix. Such an approach is highly suited to distinguishing between mono-fractal signals and general forms of multi-Hölder regularity. However, it cannot be used to discriminate between self-regulating and other forms of multi-Hölder surface, for example, which is the focus of this study. An algorithm for this class of problem was developed by Keylock (2017) and was termed the iterated, amplitude adjusted wavelet transform (IAAWT) method. It was integrated into a gradual reconstruction framework by Keylock (2018) and is described below.

\subsection{The IAAWT algorithm and gradual reconstruction}

The IAAWT algorithm is based on a dual-tree complex wavelet transform (DTCWT) (Kingsbury, 2001; Selesnick et al., 2005). A pair of dyadic wavelet trees may be constructed to form a Hilbert pair (Selesnick, 2002), resulting in a complex transform. In this paper we use symmetric, biothorgonal filters with support widths of 13 and 19 values for the first level of the algorithm and $Q$-shift filters with a support of 14 values for all other levels on the dual tree (case $\mathrm{C}$ in Kingsbury (2001)). The $Q$-shift dual tree approach retains properties that make undecimated transforms advantageous for use in surrogate generation, such as shift invariance, but at a computational cost that is merely double that for a standard discrete wavelet transform. In addition, although we do not make use of this property in this study, the transform also has enhanced directional selectivity compared to a classic discrete wavelet transform.

The IAAWT algorithm for a DEM containing elevations, $z(x, y)$, where $x=$ $y=2^{J}$, and where $J$ is an integer, proceeds as follows:

1. Store the original elevations $z(x, y)$;

2. Apply the two-dimensional DTCWT and obtain wavelet amplitudes, $A_{k, \ell, j, p}$ and wavelet phases, $\omega_{k, \ell, j, p}$ over all $j=1, \ldots, J$ scales for the $p=1, \ldots, 6$ planes at each scale and for wavelet coefficient, $w$, with coordinates, $(k, \ell)$, where at each $j$ there are $6 \times 2^{2(J-j)}$ coefficients:

$$
\begin{aligned}
& A_{k, \ell, j, p}=\left|w_{k, \ell, j, p}\right| \\
& \omega_{k, \ell, j, p}=\tan ^{-1} \frac{\mathfrak{I}\left(w_{k, \ell, j, p}\right)}{\mathfrak{R}\left(w_{k, \ell, j, p}\right)},
\end{aligned}
$$


where $\mathfrak{I}$ is the imaginary part and $\mathfrak{R}$ is the real part of the wavelet coefficients, $w$;

3. Randomly sort the original elevations to give an initial elevation surface, $z^{(0)}$

4. Take its two-dimensional DTCWT to derive randomised wavelet phases, $\omega_{k, j}^{(0)}$ for each scale and position;

5. Produce new $w_{k, j}^{(1)}$ by combining the original amplitudes with the randomised phases:

$$
w_{k, j}^{(1)}=A_{k, j} \exp \left(i \omega_{k, j}^{(0)}\right)
$$

6. Iterate the following steps until a convergence criterion is met, where at each step, $s$ :

(a) Take the inverse DTCWT to give a new DEM, $z^{(s)}(x, y)$ and then apply the amplitude adjustment step where a mapping is established between the original elevations, $z(x, y)$, and the $z^{(s)}(x, y)$ by rankorder matching to permit the values of $z^{(s)}$ to be replaced by the value in $z(x, y)$ with the same rank;

(b) Take the DTCWT and obtain the new phases, $\omega_{k, j}^{(s)}$. Combine these with the original amplitudes, $A_{k, j}$ to give the $w_{j, k}^{(s+1)}$ using the $s^{\prime}$ th iterated variant of eq. (6).

Following this approach, we generate synthetic surfaces with the exact same set of elevation values (but randomly distributed in space) and with the pointwise Hölder regularity fixed. Example surfaces are shown in Fig. 3 where the reported root-mean-squared differences are not errors as such - they indicate the degree of randomisation taking place in a given DEM. This is greatest when the central ridge becomes inverted in panel (d). A similar figure produced using a Fourier-amplitude preserving method and a statistical comparison of the two algorithms is provided in the Appendix.

Gradual multifractal reconstruction (GMR) (Keylock, 2018) generates synthetic data based on the IAAWT algorithm between limits of $\eta=0$ (corresponding to realisations using the original IAAWT algorithm) and $\eta=1$ (the original dataset). We first define an energy measure that needs to account for the decimated nature of the dual tree complex transform by weighting the coefficients by a factor $2^{j}$ (i.e. we adopt an L1 norm):

$$
E_{\eta}=\sum_{j=1}^{J} \sum_{p=1}^{6} \sum_{k=1}^{K} \sum_{\ell=1}^{L} \frac{\left|w_{k, \ell, j, p}\right|^{2}}{2^{j}}
$$

That is, with $j=1, \ldots, J$ scales, there are $K \times L$ coefficients, where $K, L=2^{J-j}$ in each of six orientation planes at each scale, meaning that more energy will be associated with each coefficient on average at the larger $j$, necessitating the introduction of the denominator. We then place the absolute values for the $w_{k, \ell, j, p}$ 

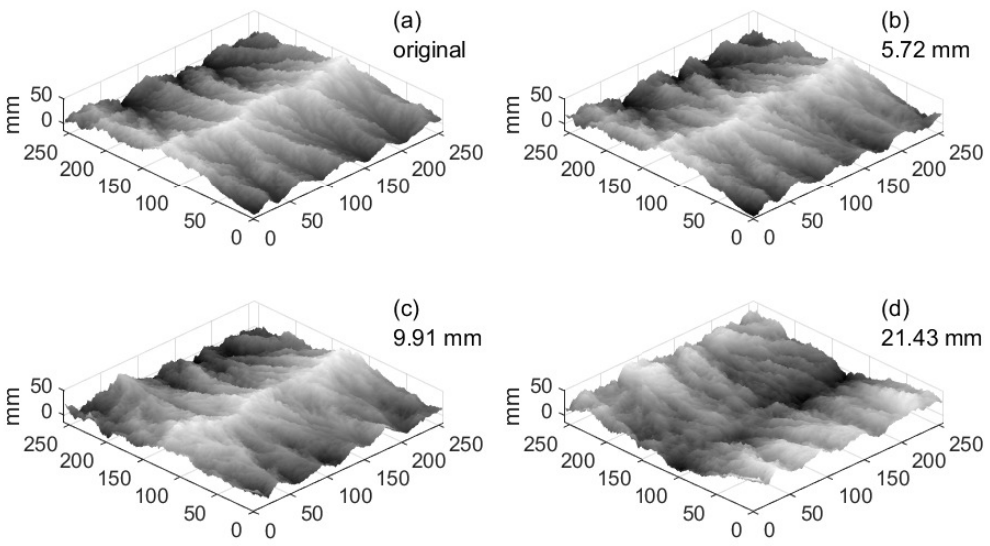

Figure 3: The original DEM at $t=500$ minutes is shown in (a), while (b), (c) and (d) show three surrogate DEMs for this surface generated with the IAAWT algorithm with the minimum, median and maximum root-mean-squared differences in the elevations, $z$, of 19 surrogates.

in descending rank order and fix the first $r$ coefficients such that $\frac{\sum_{r=1}^{K \times N}\left|w_{r}\right|^{2}}{E_{\eta}} \geq \eta$. This selected set of coefficients are fixed in place on the wavelet coefficient template, while the others are phase randomized using eq. (6). More coefficients can be fixed until there is no significant difference between the original data and the surrogates with respect to a chosen metric to characterize the surfaces and, thus, establishing a threshold value for $\eta$, which is denoted by $\eta^{*}$ in this manuscript. A higher value for $\eta^{*}$ implies greater additional complexity in the data, beyond the preservation of the Hölder regularity.

Using the IAAWT surrogate data algorithm within the GMR framework, and given a chosen measure for geomorphometric similarity (as described in the next sub-section), the form for the null hypotheses in this paper is as follows: Landscape system complexity with respect to a chosen metric of geomorphometric similarity is inferred by statistical comparison between the source DEM and the surrogate landscapes that preserve the elevations and multi-Hölder structure of the original DEM (at the $\eta=0$ limit), as well as an increasing proportion of other properties of the DEM as $\eta$ increases.

\subsection{Geomorphometric measures}

In this study, we draw upon three basic classes of geomorphometric analysis. The first is based on the slope-area relation, which Willgoose (1994) showed was highly relevant to the study of evolving topographies with both tectonic and climatological forcing. It should be highlighted that we investigate drainage basin slope-area scaling as is common in small-scale experimental studies (Lague et al., 

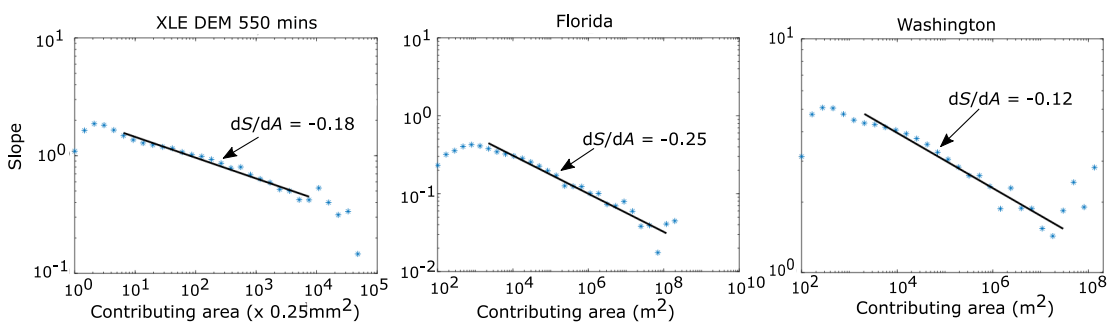

Figure 4: The nature of the scaling between basin slope and contributing area for the experimental data at $t=550$ minutes (top-left), the Florida DEM (top right) and the Washington DEM (bottom left). The length of the line indicates the limits for the fit applied to the original and surrogate DEMs.

2003; Bonnet and Crave, 2006), rather than channel slope-area scaling, which is more common in field scale analyses of mature topography where identifying channels is rather more straightforward (Kirby and Whipple, 2012). The consequence is that our exponent, $d S / d A$ has a lower value as in headwater basins both slopes and channels are steep, while larger basins tend to have very low gradient river channels, but still a mix of hillslope gradients.

We computed topographic slopes using the steepest downslope direction whereas the drainage areas were determined using the D-infinity algorithm (Tarboton, 1997; Dietrich and Perron, 2006). Slope-area curves were computed based on averaged topographic slopes in equally spaced logarithmic bins representing drainage area. Although, in general, three regimes were identified in the slope-area curve of the experimental landscapes at the steady state (Singh et al., 2015), here we focused on the longer (intermediate) log-log linear regime that exhibited a scaling exponent of $\sim 0.1$. This low scaling exponent has been argued to be a characteristic of colluvial landscapes that are dominated by debris flows and mass wasting events (Montgomery and Foufoula-Georgiou, 1993; Montgomery, 2001; Bonnet and Crave, 2006). Note that similar scaling exponents were observed from other physical experiments (Hasbargen and Paola, 2003; Lague et al., 2003; Bonnet and Crave, 2006). Also note that, for computing scaling exponents for DEM surrogates, we used the same scaling range as that of the original DEM.

Figure 4 shows example fits of the slope-area scaling for one of our experiments from the experimental landscape evolution facility at $t=550$ minutes, as well as for the Florida and Washington DEMs examined later in this paper. In all cases, the power-law scaling exists over at least two orders of magnitude with relatively low variance indicating that the exponent is estimated well. Statistical significance to the results is then ascribed using the surrogate data. I.e. given the fits are precise, we focus on if there is a statistically significant difference in the exponent between data and multi-Hölder preserving surrogates as a function of $\eta$, rather than on the fitting of the exponents. This is an advantage of our approach - we focus on adopting a consistent means for estimating the 
exponent and the significance via the surrogates and $\eta^{*}$ rather than the exact value for the exponent. The consequence is that we can place less emphasis on the subtleties that can arise when focusing on the exact value for the exponent, as discussed recently by Gailleton et al. (2019).

The second class of geomorphometry utilizes the notion of Horton-Strahler channel ordering to classify sub-catchments in the DEM into different basin orders, $\Omega$. To derive the channel in the DEM we used topotoolbox (Schwanghart and Kuhn, 2010) with a threshold area of 100 pixels and a D8 flow accumulation to obtain the channel heads numerically. This corresponded to a drainage area of $25 \mathrm{~mm}^{2}$ for the experimental DEMs and $10^{4} \mathrm{~m}^{2}$ in natural DEMs. For all of the basins of a given order, we then derived the number of basins at that order, $N_{B}(\Omega)$, and the average total channel length for a given order, $\left\langle\sum L(\Omega)\right\rangle$. Typically, power-law relations for these quantities are found (Rodgriguez-Iturbe and Rinaldo, 1997). However, here we focus on the raw values rather than the fitted exponent to contrast with the approach taken with slope-area scaling. These first and second class of geomorphometric measures are similar to those adopted in related work (Singh et al., 2015; Tejedor et al., 2017). Our third method is based on a recently proposed variant of terrain hypsometry (Strahler, 1952), but modified to include simultaneous consideration of the Hölder regularity of a landscape (Keylock et al., 2020b). Rather than look at the condtional hypsometric curves as function of $h$, as we did in our recent paper, here we simply focus on the joint distribution function of the elevations and Hölder exponents. In other words, we examine if there is structure to the coupling between elevation and terrain roughness that a multi-Hölder model cannot capture.

For the analysis of the experiment we employed seven $\eta$ values $(\eta \in\{0.0,0.2,0.4,0.6,0.8,0.9,0.99\})$ and generated 19 surrogate DEMs for each of twenty-two experimental DEMs using GMR (i.e. 22 original and 2,926 synthetic DEMs were analyzed). These spanned the times from when the drainage basin was first established at $t \sim 150$ minutes, through the attainment of a sediment flux equilibrium at $t \sim 350$ minutes, to beyond the development of a morphometric steady-state at $t \sim 375$ minutes.

\section{Results}

\subsection{Slope-area relations}

Figure 5 shows how the threshold values, $\eta^{*}$ are determined for the slope-area scaling, $d S / d A$, for DEMs obtained at four instances that span the experimental duration. All DEMs clearly have values for $d S / d A$ that depart from those obtained from a simple multi-Hölder representation of the terrain at $\eta=0$. Once the central drainage divide is established in the experiment, i.e. for $t \gtrsim 30$, the surrogate landscapes differ from the actual DEM for $\eta \leq 0.4$. With 19 surrogates generated and a directional, one-tailed hypothesis that $d S / d A$ for the surrogates is not significantly greater than for the original DEM, our analysis indicates a significant difference at the $5 \%$ level up to $\eta=0.8$ for most cases. 

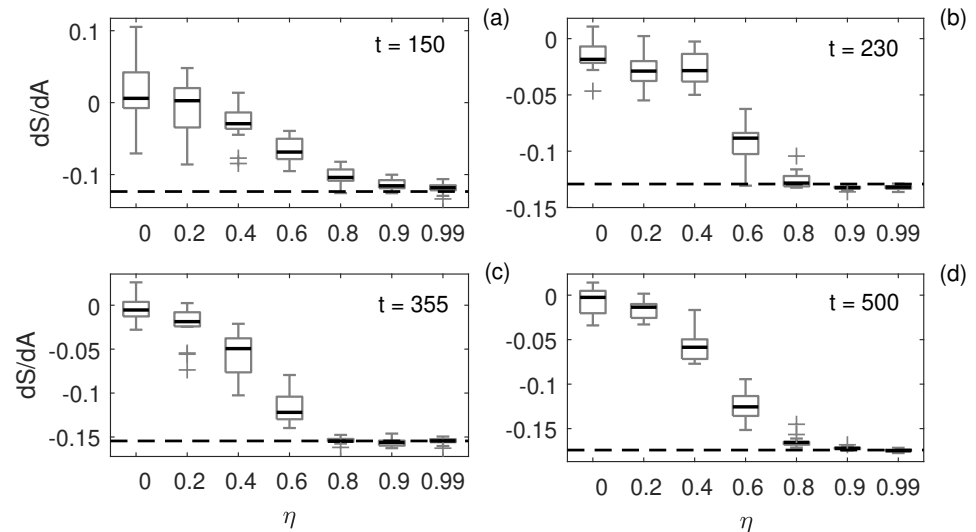

Figure 5: Boxplots showing values of $d S / d A$ for the original DEM (horizontal, dashed line) and surrogates (boxplots) as a function of $\eta$. The threshold, $\eta^{*}$ is obtained by working from right to left until the last case is found for which there is no signficant difference between data and surrogates. This gives $\eta^{*}=$ $\{0.8,0.6,0.8,0.9\}$ for $t=150, t=230, t=355$, and $t=500$ minutes, respectively. The boxplots are formulated in the same way as in Fig. 18.

Given values for $\eta^{*}(d S / d A)$ for all 22 DEMs, Fig. 6b shows how these vary as a function of the evolution of the catchment. All values are high and there is a possible weak tendency in these results with random variability in the range $0.6<\eta^{*}(d S / d A) \leq 0.9$ up until a flux equilibrium is established at $t \sim 350$ minutes, followed by a gradual increase in $\eta^{*}(d S / d A)$ beyond this point. Figure 6a shows the values for $d S / d A$ as a function of time, with a quadratic decay illustrated by a red line. The vertical lines extending from each symbol show a form of confidence interval that is possible using surrogate data analysis (Keylock, 2012); one based on the range of values for the surrogates at the appropriate $\eta^{*}(t)$, rather than quality of fit to one set of data. It is clear that: (a) the one instance of $\eta^{*}(d S / d A)=0.6$, at $t=225$ minutes, is a consequence of the wide range of values for $d S / d A$ obtained for the surrogates at this time; and, (b) the change in $d S / d A$ with time is significant.

Given there is a possible change in behavior of landscape features and processs after the flux equilibrium is attained, we examined the proportion of wavelet coefficients fixed for this DEM. The observed value for $\eta^{*}=0.8$ arises when $0.34 \%$ of the coefficients are fixed (1787 from 524286 coefficients). For $\eta=0.6$ this drops by nearly an order of magnitude to $0.04 \%$, while for $\eta=0.9$ and $\eta=0.99$, the percentages are $1.4 \%$ and $14.7 \%$, respectively. These percentages increase by $20 \%-30 \%$ at $t=500$ minutes, highlighting how the increase in landscape complexity is not just measured by an increase in $\eta^{*}$ but also consistent increase in the fixed coefficients for a given $\eta$. Comparing the results for $t=230$ minutes to those at $t=350$ minutes, the number of fixed coefficients at 

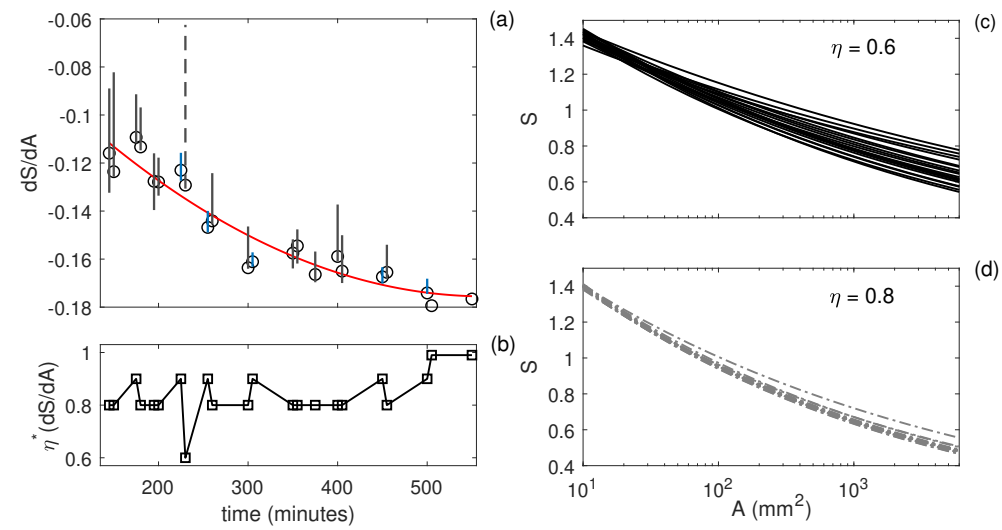

Figure 6: The slope-area scaling exponent as a function of time, $t$, is shown in panel (a) as a circle, with a best-fit quadratic as a red curve. The vertical dark gray lines show the range of values for the gradual multifractal reconstruction (GMR) surrogates at the value for $\eta^{*}(d S / d A)$, with $\eta^{*}(d S / d A)=0.6$ (dashed, gray), $\eta^{*}(d S / d A)=0.8$ (solid, gray), and $\eta^{*}(d S / d A)=0.9$ (solid, blue). The variation for $\eta^{*}(d S / d A)=0.99$ is no greater than the diameter of the circles and is not shown. Panel (b) shows these values for $\eta^{*}(d S / d A)$ as a function of time. Panels (c) and (d) show the relation between basin slope and upstream contributing area for the surrogate data at $t=500$ minutes for $\eta=0.6$ (c) and $\eta=0.8$ (d). Results are shown on semi-logarithmic axes for clarity, although all fits are of a power-law form. 
a given $\eta$ is about a third, indicating that these landscapes are simpler than those at the end of the experiment both because the value for $\eta^{*}$ is lower and because fewer coefficients need to be fixed for a given proportion of the wavelet energy. Thus, from $t=230$ to $t=350$ minutes complexity in the underlying DEM certainly increases greatly, even if the threshold for statistically detecting convergence on the DEM's properties $\left(\eta^{*}\right)$, is similar.

(a)

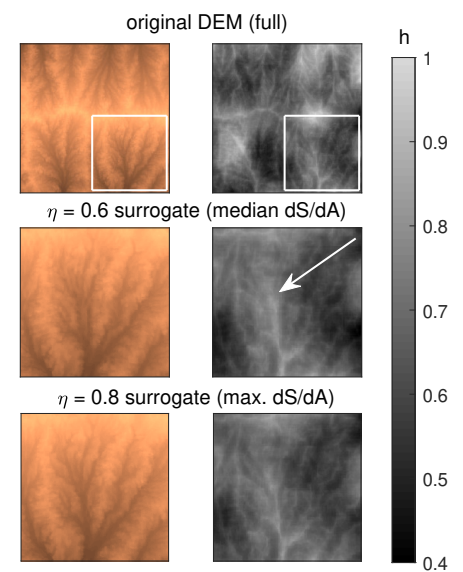

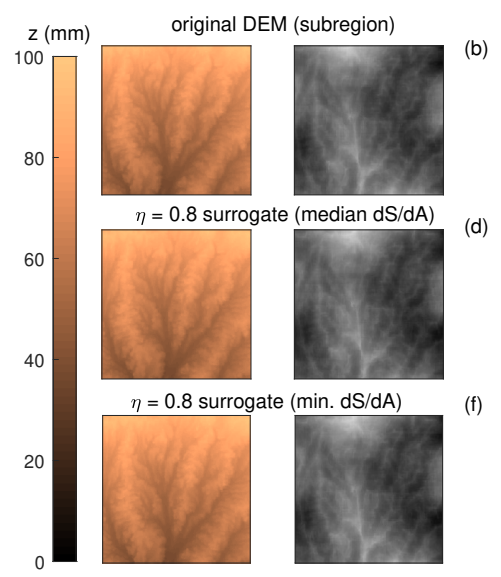

Figure 7: The DEM extracted at $t=500$ minutes in plan view, together with accompanying surrogate data. In each of six cases, two panels are shown with the left-hand cases (copper color scheme) illustrating the elevations, $z$, and the right-hand (gray color scheme) the Hölder exponents. Panel (a) is the original DEM with a white box showing the region extracted in the other panels. Panel (b) is this extracted region, (c) is the surrogate DEM at $\eta=0.6$ with the median value for $d S / d A$, while (d), (e) and (f) are the surrogate DEMs at $\eta=0.8$ with the median value, maximum and minimum values for $d S / d A$, respectively. The arrow highlights a feature discussed in the text.

In order to understand why the $\eta^{*}(d S / d A)$ values are generally high, in Fig. $6 \mathrm{c}$ and $\mathrm{d}$ we plot the fitted power-laws for the surrogates using the DEM for $t=$ 500 minutes as an example. For $\eta=0.6$ and $\eta=0.8$ the slopes for the small area basins are the same $(\sim 1.4)$, while they are significantly lower for the large basins at $\eta=0.8$. Hence, we can explain why the surrogates cannot replicate the $d S / d A$ values at low $\eta$ : the slopes of the larger basins are too steep. As small basins are typically located in the headwater catchments, the true landscape has a stronger coupling between lower elevations and reduced gradients, implying some degree of self-regulation as considered in section 4.4. Thus, the shortcomings of a simple multi-Hölder model are less in the representation of the dissected, upper basins but rather in the correct representation of the deposition-dominated, larger catchments.

To illustrate the difficulty of detecting these differences by observation and, thus, the difficulty of evaluating the verisimilitude of a multi-Hölder model by 
qualitative assessment, we show in Fig. 7 the detail of the original data and various surrogate DEMs for $t=500$ minutes. Panel (a) shows a plan view of the original DEM, while panel (b) highlights the region in the white box in panel (a). From Fig. 5d, the surrogate DEM at $\eta=0.6$ with the median value for $d S / d A$ and that at $\eta=0.8$ with the maximum for $d S / d A$ shown in panels (c) and (e), respectively, are in error, while the surrogate at $\eta=0.8$ with the median and minimum for $d S / d A$ shown in (d) and (f), respectively, are very close to the original case. The highlighted area of the DEM is one containing a large basin consisting of low elevations and high values for $h$. The primary visible difference is that the spine of high $h$ values in (c) indicated by the arrow is too broad and diffuse relative to the cases seen in panels (b), (d) and (f). This results in high $h$ values being associated with somewhat higher $z$ values than in the original DEM, causing reduced values for $d S / d A$ at $\eta=0.6$ compared to the true case. Clearly, a surrogate data framework is needed to extract such subtleties with statistical confidence.

\subsection{Average total channel length}
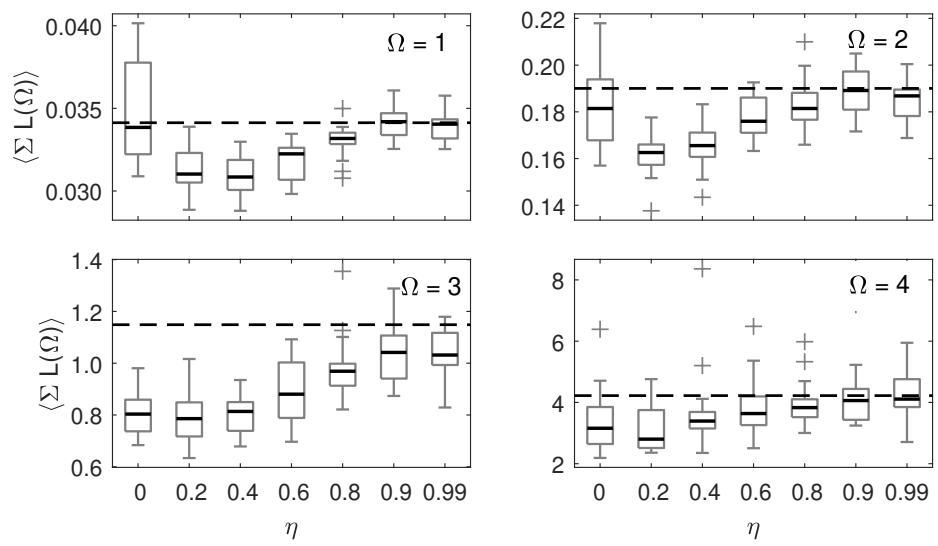

Figure 8: Boxplots showing the determination of $\eta^{*}(L)$ for the DEM at $t=$ 150 minutes for basin orders $\Omega \in\{1,2,3,4\}$. The values of $\left\langle\sum L(\Omega)\right\rangle$ for the surrogates are shown as boxplots a function of $\eta$, with the dashed lines showing the value for the data itself. From these plots, $\eta^{*}(L)=\{0.8,0.6,0.8,0.0\}$ for ascending values of $\Omega$. The boxplots are formulated in the same way as in Fig. 18.

Figures 8 and 9 show the average channel length for basins of four HortonStrahler orders at two different times as a function of $\eta$. These results contrast with the slope-area scaling as a simple multi-Hölder model with no additional constraints $(\eta=0)$ can replicate the observed channel lengths in many cases, even though $\eta^{*}$ itself is often greater. Furthermore, in neither case do the fourth order basins indicate any significant differences. These are the largest in 

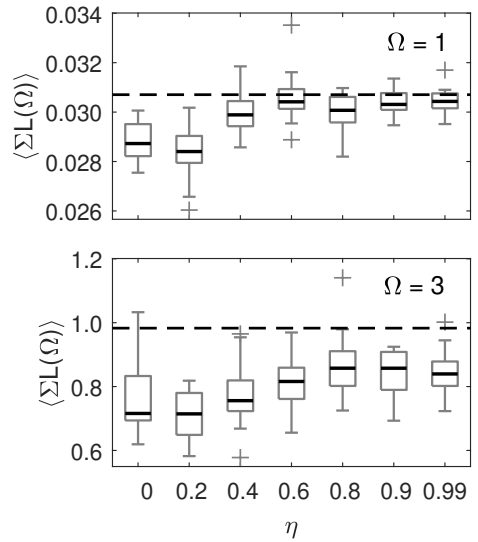
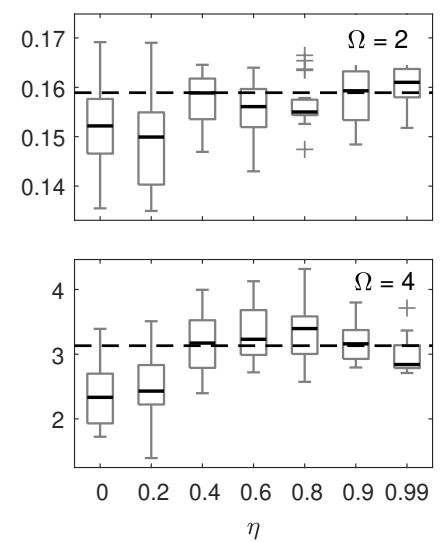

Figure 9: Boxplots showing the determination of $\eta^{*}(L)$ for the DEM at $t=$ 500 minutes for basin orders $\Omega \in\{1,2,3,4\}$. The values of $\left\langle\sum L(\Omega)\right\rangle$ for the surrogates are shown as a function of $\eta$, with the dashed lines showing the value for the data itself. From these plots, $\eta^{*}(L)=\{0.4,0.0,0.99,0.0\}$ for ascending values of $\Omega$. The boxplots are formulated in the same way as in Fig. 18 .

the system and are integrating information over a sufficiently large area that the preservation of the elevations and the approximate preservation of the $h$ is sufficient to get the average channel length statistics correct. However, at $t=150$ minutes and before the drainage divide is firmly established, the values of $\left\langle\sum L(\Omega)\right\rangle$ for $\Omega=3$ are a sensitive measure, implying that the properties of third order basins are highly dependent on the structure of the main divide. While $\eta^{*}$ is also high for $\Omega=3$ for $t=500$ minutes, the $\eta=0$ case can attain the requisite values by chance. In contrast, in this case, it is the $\Omega=1$ basins where additional structure is required to get the correct channel length statistics, with the surrogates producing lengths that are too short. The implication of the slopes being matched very well for small basins at $t=500$ minutes (Fig. 6c) but $\left\langle\sum L(\Omega=1)\right\rangle$ being too small is that a simple multi-Hölder model cannot adequately represent either basin shape or valley sinuosity effects correctly.

\subsection{Total number of basins}

Three classical scalings for drainage basins as a function of stream order are the laws of stream number, stream length and basin area (Rodgriguez-Iturbe and Rinaldo, 1997). Because we found that the behavior for basin area was similar to that for stream length, we do not report those results here, focusing instead on the stream number results, which in terms of our analysis we state as the number of basins of a given order, $N_{B}(\Omega)$ and present the results for $t=500$ minutes in Fig. 10. Here a much stronger effect was found in terms of significant differences at $\eta=0$ for different stream orders less than $\Omega=4$. Thus, $N_{B}(\Omega)$ is a more sensitive metric than $\left\langle\sum L(\Omega)\right\rangle$ for studying landscape complexity. In Fig. 

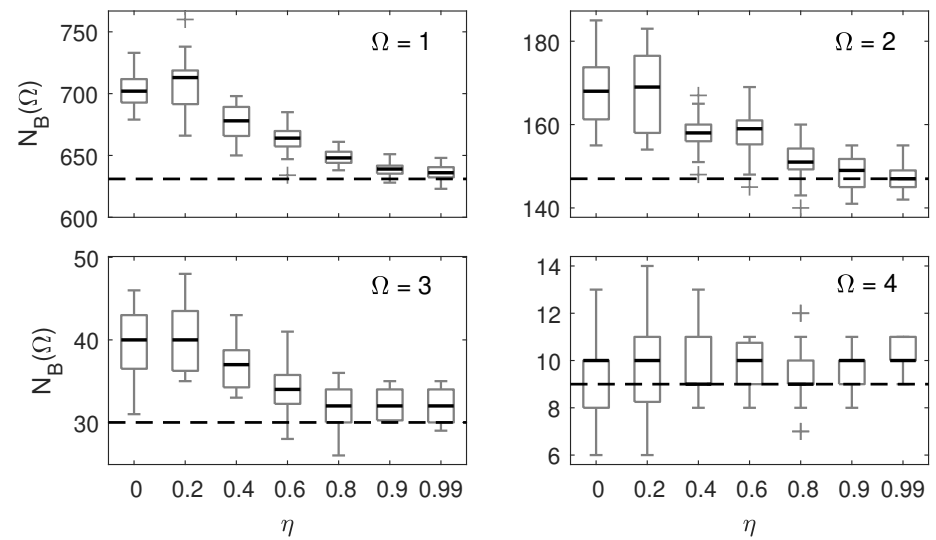

Figure 10: Boxplots showing the determination of $\eta^{*}\left(N_{B}\right)$ for the DEM at $t=500$ minutes for different basin orders, $\Omega$. The values of $N_{B}$ for the surrogates are shown as a function of $\eta$ in each panel, with the dashed lines showing the value for the data itself. From these plots, $\eta^{*}\left(N_{B}\right)=\{0.9,0.6,0.6,0.0\}$ for stream orders, 1 to 4 , respectively. The boxplots are formulated in the same way as in Fig. 18.

11 we plot $\eta^{*}\left(N_{B}\right)$ for all four stream orders as a function of time. As with the results for $d S / d A$ in Fig. 6b, a transition seems to emerge after about $t=350$ minutes, but the effect is much more marked here: complexity measured by $\eta^{*}$ really only increases once the flux steady-state is established for $t>350$ minutes; it is only in the early stages of landscape that a simple multi-Hölder model is effective. Given that flux steady state was defined as the condition where the erosional fluxes balanced out the sediment provided by the rock uplift and was obtained by direct measurement during the experiment (Singh et al., 2015), the congruence between the attainment of this state and the increase in $\eta^{*}$ is rather remarkable. The implication is that complexity increases once diffusive forces gain greater prominence in the landscape dynamics, although without processbased measurements one cannot disregard the possibility that the nature of the diffusive processes themselves have a more marked impact on the nonlinear response of the terrain at later times.

\subsection{The joint distribution of elevation and regularity}

A recent extension to the classic hypsometry measure of Strahler (1952) (see Leuven et al. (2018) and Cunningham et al. (2019) for recent uses of hypsometry) examines the joint probability distribution function (PDF) between the elevations and the Hölder exponents (Keylock et al., 2020b). In other words, it captures the coupling that underpins the nature of a self-regulating landscape as discussed in the introduction. The top-left panel of Fig. 12 shows this PDF for the original DEM at $t=500$ minutes. The other panels show the results for 


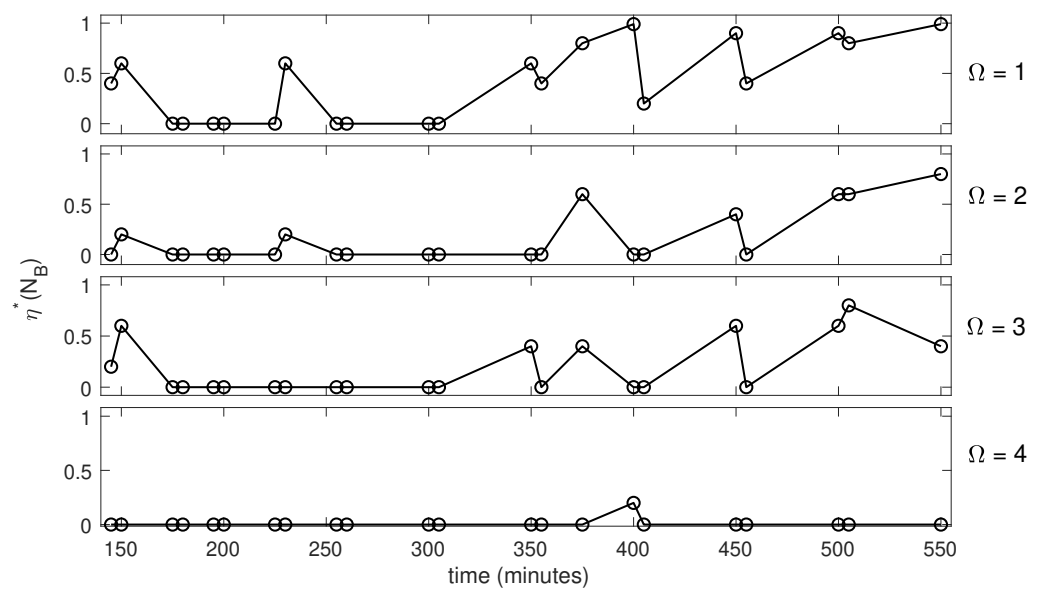

Figure 11: Values for $\eta^{*}\left(N_{B}\right)$ as a function of stream order, $\Omega$ (each panel) and time.

the surrogate DEM with the median RMSE for different choices of $\eta$. Recall that our algorithm uses exactly the same $z$ values. Hence, there is no difference in the marginal distribution for $z$ and the hypsometries for all of these data are identical. However, there are clear differences in the shape of the joint PDFs and, as $\eta$ increases, relatively subtle features of the original PDF, such as the outlying region identified by the arrow in the top-left panel, begin to be captured in the surrogates. Here we focus on two conditional distributions given by the transects through the joint distribution shown by the dashed lines in the upper left panel. These pass through the mode of the distribution at $z=25$ $\mathrm{mm}, h=0.95$ and are given by the black line in each panel of Fig. 13. The gray lines in this figure are the equivalent conditional distributions for the surrogates at the stated value for $\eta$. It is clear that at low $\eta$, the surrogate data cannot replicate this mode, which is too large in magnitude for $p(z \mid h=0.95)$ given in the right-hand column and is located at too high a value for $h$ for $p(h \mid z=25)$ in the left-hand column. A threshold value of $\eta^{*}=0.4$ is appropriate from this analysis. Hence, once more, a simple multi-Hölder model cannot serve, and the key difficulty for such a model in terms of self-regulation is to have a sufficient number of intermediate elevations that are as smooth as $h=0.95$.

Physically, this means that diffusive processes, which increase $h$ at intermediate elevations, gain in geomorphic significance either in terms of intensity or the area dominated by diffusion once the landscape attains a flux equilibrium, and are more important to the landscape structure than a simple multi-Hölder model can capture. This result is consistent with the earlier results that the simple multi-Hölder model produces too many basins of a given order (incision is excessive relative to diffusion) and has slopes that are over-steepened within the largest basins. Significant diffusive action on the intermediate slopes will result in fewer basins of intermediate order and will promote a reduction in average 

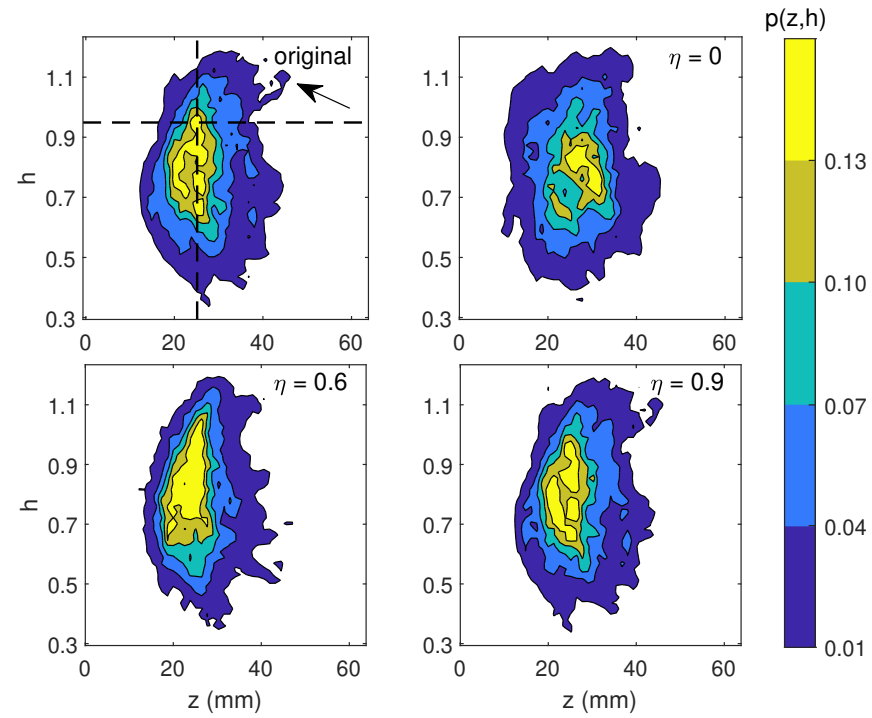

Figure 12: Probability contours for the joint distributions of the elevations, $z$, and Hölder exponents, $h$, for the DEM obtained at $t=500$ minutes for the original data (a) and three surrogate datasets at the value for $\eta$ stated in each panel. Each surrogate DEM shown is that with the median RMSE between data and surrogates for the joint PDF. The dashed construction lines in panel (a) are the transects examined in Fig. 13 and the arrow identifies a feature discussed in the text.

slopes for the larger watersheds of which these slopes are a part. The potential to use the Hölder regularity of the landscape to detect transient effects such as over-steepened slopes was looked at by Keylock et al. (2020b) who applied an anisotropic diffusion operator to the terrain that moved mass in the DEM towards regions of strong local curvature. While this operation had a minimal impact on the hypsometry, it impacted on the Hölder regularity such that it was easy to detect the effect on the terrain, even without employing the surrogate data methodology developed in this paper.

\section{Application to two distinct topographies}

While the experimental surfaces allow us to examine the evolution of a topography's response to a particular forcing, the idealized boundary conditions, particularly the issue of material homogeneity, mean that there is not necessarily a relation to any specific observed terrain. As a consequence, in this section of the paper we examine the geomorphometry of two contrasting regions of the conterminous United States, Florida and Washington State, focusing on the 

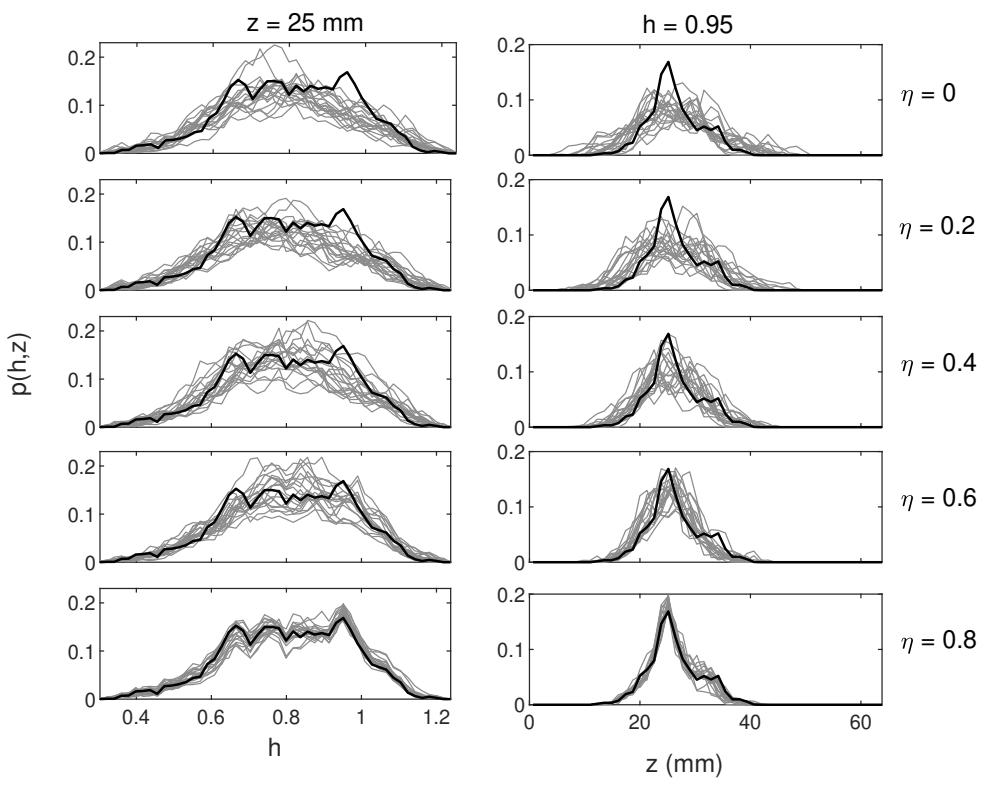

Figure 13: Transects through the joint PDF shown in Fig. 12a are shown in each panel as a solid line, together with the transects for nineteen surrogates at the stated value for $\eta$. The left-hand column of panels show the transect, $p(h \mid z=25$ $\mathrm{mm}$ ), i.e. the vertical line in Fig. 12a), while the right-hand column is for the horizontal line in Fig. 12a, i.e. $p(z \mid h=0.95)$. The conditional distributions shown are not renormalized; they are transects through the joint PDF.

slope-area scaling properties. The two DEMs were obtained from the USGS National Elevation Dataset at https://catalog.data.gov/dataset/usgs-nationalelevation-dataset-ned and both cover an area of $20.48 \times 20.48 \mathrm{~km}$ at a $1 / 3$ arcsecond $(10 \mathrm{~m})$ resolution. The particular drainage basins are the Ochlockonee River basin in Florida and the Cowlitz River basin in Washington State and these are shown in Fig. 14. The elevation range in the former basin is $0.5 \mathrm{~m}$ to $105 \mathrm{~m}$, and is $263 \mathrm{~m}$ to $4100 \mathrm{~m}$ in the latter. Within the extracted regions that are the focus of our investigation, the elevations range from $33 \mathrm{~m}$ to $101 \mathrm{~m}$ for the Florida case, and $361 \mathrm{~m}$ to $2274 \mathrm{~m}$ for the Washington case. The natural DEMs were projected in a metric system and the topographic depressions (e.g. sinks) were filled to enable continuous flow. Thereafter, processing followed that used for the experimental data.

Panels (a) and (b) in Fig. 15 show that the slope-area scaling for these two topographies is significantly different, with the scaling exponent nearly double in magnitude for the Florida case. The value for Florida of $d S / d A=0.25$ is tending towards the values seen for channel slope-area relations in fluvial landscapes (Montgomery, 2001; Lague et al., 2003; Hooshyar et al., 2017). Clearly, as the scale of the system increases, the basin slope-area scaling we have used for 

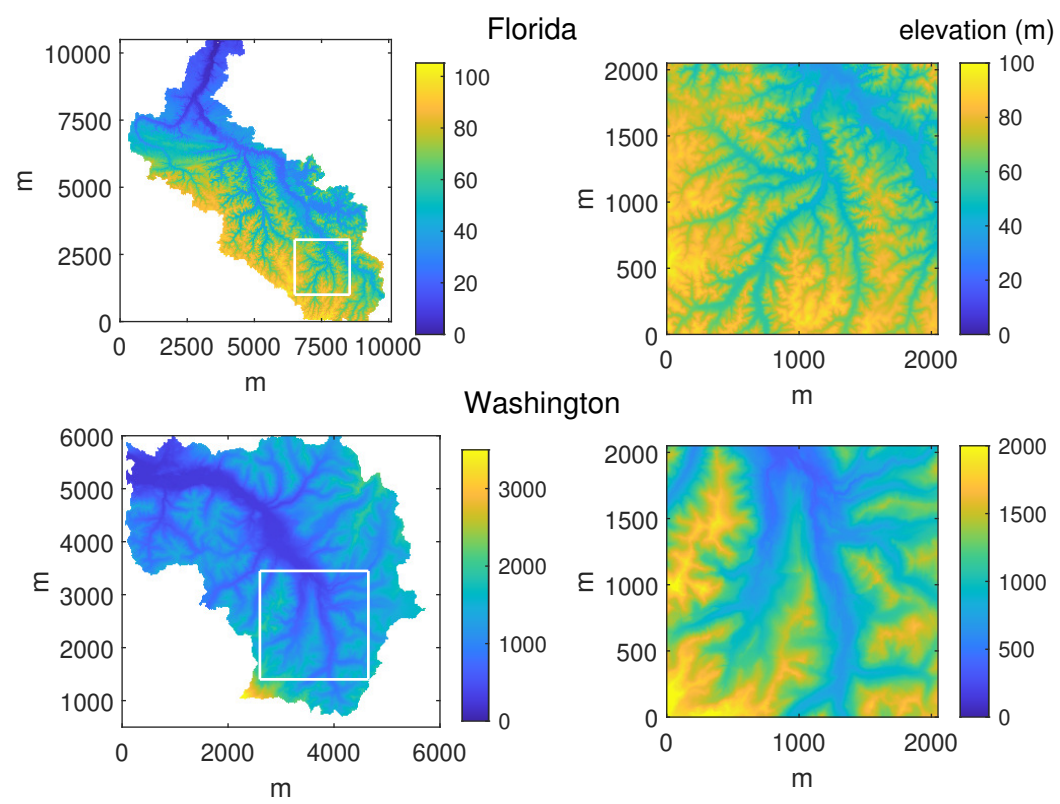

Figure 14: The two drainage basins from Florida (upper) and Washington State (lower) are shown in the left-hand panels, with the $2048 \times 2048 \mathrm{~m}$ sub-regions that are analyzed in detail highlighted in the right-hand panels. The colorbars show the elevation range $(m)$ in each panel.

consistency with the experimental results is converging towards the values anticipated for channel slope-area scalings (Kirby and Whipple, 2012). The Washington data retains a shallower value of $d S / d A=0.18$ as a consequence of the stronger influence of mass-wasting and colluvium-dominated processes. Despite these differences, both basins have $\eta^{*}=0.999$, although while the surrogates for the Washington DEM have a slope-area scaling exponent that converges on the true values in an approximately linear fashion, the Florida data exhibit a rapid decrease in the value of $d S / d A$ from $\eta=0.2$ to $\eta=0.5$. The largest change in the $d S / d A$ values for the Washington data occurs from $\eta=0.99$ to $\eta=0.999$. To investigate this further we examined the fixed wavelet coefficients at these choices for $\eta$. The proportion of fixed coefficients as a function of $1-\eta$ (to apply a logarithmic scale more easily) is given in Fig. 15c. It is notable that for both DEMs, $\eta=0.999$ equates to about $20 \%$ of the coefficients being fixed (left-most dotted line), but that for lower values for $\eta$ (to the right in this panel) there is a clear divergence in the proportion of coefficients fixed between the two DEMs, with energy spread among a greater number of coefficients for the Florida case. To understand these results more clearly, we found the difference in the elevations for the DEMs reconstructed from the fixed wavelet coefficients only for the 

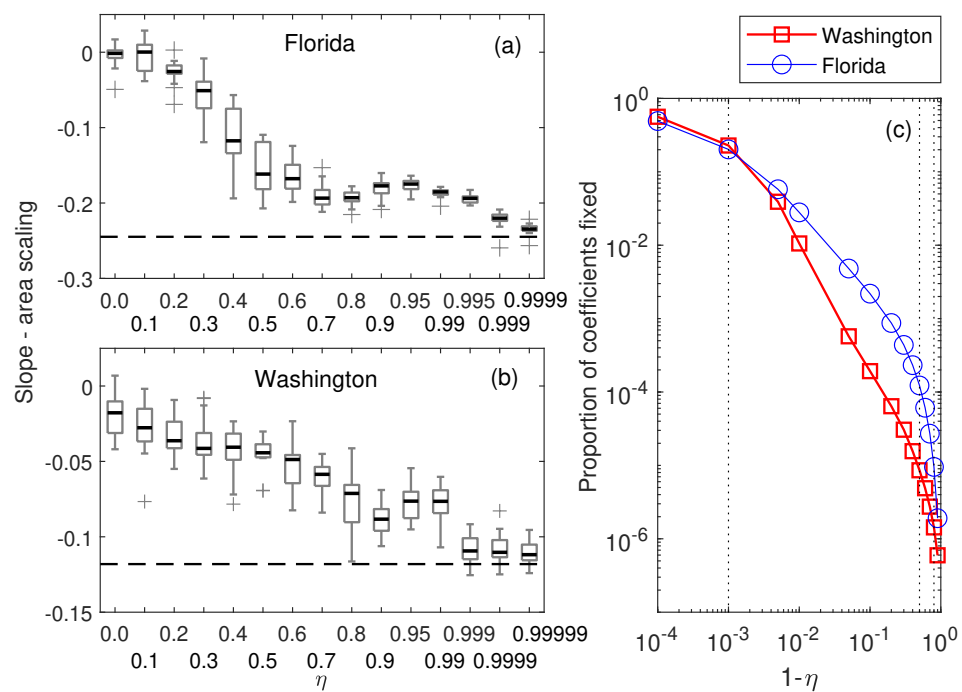

Figure 15: Values for the slope-area scaling exponent for the surrogate data as a function of $\eta$ for Florida (a) and Washington (b), with the actual value shown as a horizontal, dashed line. Panel (c) shows the proportion of fixed wavelet coefficients as a function of $1-\eta$ for the Washington and Florida DEMs. Vertical dotted lines highlight the values at, from left to right, $\eta=0.999, \eta=0.5, \eta=0.2$ as discussed in the text.

values of $\eta$ that represent the major changes seen in Fig. 15a and b. We then derived the joint distribution functions for these differences $(z(\eta=0.5)-z(\eta=0.2)$ and $z(\eta=0.999)-z(\eta=0.99))$ against the original elevations, which are shown in Fig. 16 together with the marginal distributions for the original elevations.

For the Florida data, the top-left panels show that the dominant change as $\eta$ increases from 0.2 to 0.5 is an increase in the elevation by $2.6 \mathrm{~m}$, concentrated at an elevation of $78 \mathrm{~m}$. The correlation between the two variables is also rather strong, $R=0.55$, indicating a clear tendency for the positive elevation differences to arise preferentially at the mid to upper elevations. Consequently, we can conclude that the key difficulty for a multi-Hölder, $\eta=0$ model in replicating the slope-area scaling for the Florida case-study is in allocating sufficient heights to the elevations at the mode of the marginal distribution for elevations in the original DEM. In other words, these regions in the terrain are dissected too much in the low $\eta$ surrogates, while in nature the greater preponderance of diffusive processes preserves these elevations. This is consistent with the earlier analysis of the number of $\Omega=1$ basins and of the slope-area scaling for the experimental surfaces. Preserving mass in the topography in the $60 \mathrm{~m}-80 \mathrm{~m}$ elevation range at $\eta=0.5$ results in greater slopes in the smaller area basins, changing the median slope-area scaling for the surrogates from -0.026 for $\eta=0.2$ to -0.162 at $\eta=0.5$. 

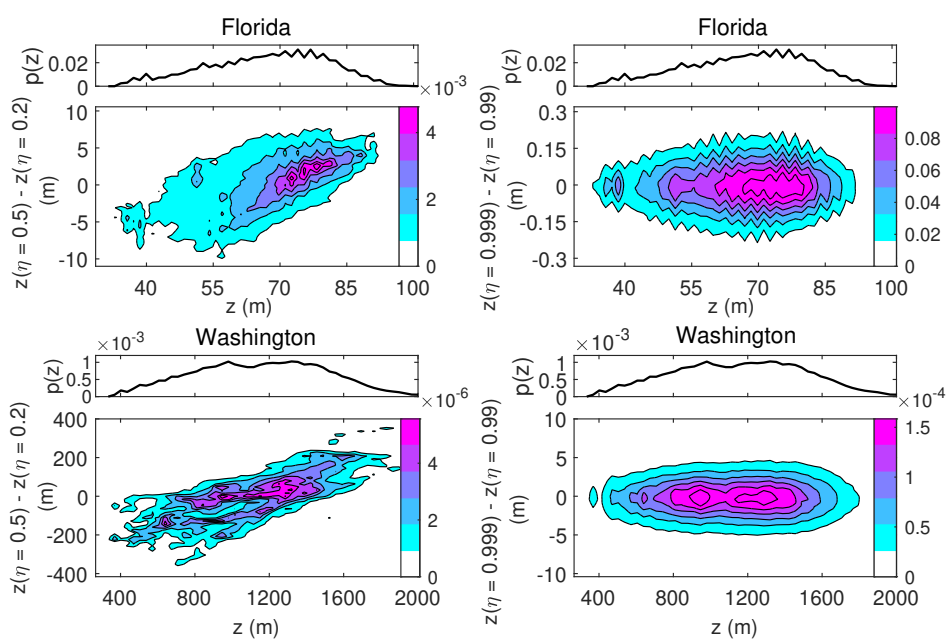

Figure 16: The joint probability distribution functions for Florida (top row) and Washington (bottom row) between the elevations in the original DEM and the difference in elevations between the DEMs reconstructed from the fixed wavelet coefficients. The marginal distributions of the elevations are shown above each main panel. The differences used are $z(\eta=0.5)-z(\eta=0.2)$ (left-hand panels) and $z(\eta=0.999)-z(\eta=0.99)$ (right-hand panels).

The tendency for a positive covariance to exist in the joint distribution at $z(\eta=0.5)-z(\eta=0.2)$ is also seen for the Washington data with $R=0.69$. However, in this case the mode is located centrally at $z=930 \mathrm{~m}, z(\eta=0.5)-z(\eta=$ $0.2)=0 \mathrm{~m}$, although there are a number of strongly expressed additional modes, such as that at $z=630 \mathrm{~m}, z(\eta=0.5)-z(\eta=0.2)=-150 \mathrm{~m}$, and $z=1600 \mathrm{~m}$, $z(\eta=0.5)-z(\eta=0.2)=200 \mathrm{~m}$. These latter modes also indicate that a multiHölder model fails to preserve the lower and upper elevations appropriately, resulting in incorrect slope-area scalings.

For both regions, the change to $\eta^{*}=0.999$ is symmetric in the difference distribution for $z(\eta=0.999)-z(\eta=0.99)$ with no structure as a function of $z$ - the joint distribution reflects the nature of the marginal distribution for $z$. Thus, the final stage of convergence to no significant difference in the slope-area scaling is simply about removing a sufficient number of small-scale irregularities. Hence, we can see that two very different landscapes exhibit qualitatively similar behaviors in terms of the manner by which the elevations are fixed in the wavelet coefficients for $0.2 \leq \eta \leq 0.5$ and then $0.99 \leq \eta \leq 0.999$. Given that in both cases $\eta^{*}=0.999$, the major difference is in the nature of the $d S / d A$ scalings in Fig. 15 . What Fig. 16 reveals about this is that the major drops towards convergence $(0.2 \leq \eta \leq 0.5$ for Florida and $0.99 \leq \eta \leq 0.999)$ arise at a similar length scale. The standard deviation of $z(\eta=0.5)-z(\eta=0.2)$ for Florida is $4.4 \mathrm{~m}$, and is $2.7 \mathrm{~m}$ for $z(\eta=0.999)-z(\eta=0.99)$. This is most likely an artifact of the 10 
$m$ resolution of the two DEM products, but does imply that GMR may be able to detect any fundamental scale-breaks in the terrain from the manner that topographic metrics change as a function of $\eta$.

\section{Discussion and Conclusion}

In this paper we have formalized the nonlinear analysis of digital elevation models using the gradual multifractal reconstruction (GMR) framework. In particular, we have used experimental, evolving landscapes to show that a simple multi-Hölder model for terrain, even with the set of elevations, $z$, constrained to the original values and the pointwise Hölder exponents located correctly in the terrain, is not sufficient to replicate several measures of geomorphometry for mature topography. Our analysis framework has shown that the slope-area scaling relation, $d S / d A$ and, particularly, the number of basins for a given HortonStrahler stream order (when this is less than the scale of the system studied) are sensitive measures of landscape structure. In fact, the number of basins for most stream orders could be replicated appropriately by a multi-Hölder model at early times, but once a sediment flux equilibrium was established at $t \sim 350$ minutes, complexity increased dramatically, as shown by the increase in $\eta^{*}$ in Fig. 11. Values for $\eta^{*}$ remained high even when the landscape appeared to be in a steady-state, with the key geomorphic insight being that diffusive processes in the experimental landscape act to reduce the number of basins relative to the statistical models. We emphasise that it is only by using the surrogate data approach that the significance of these results can be established. Hence, while the slope-area results suggest $\eta^{*}$ is always high, the $N_{B}$ results for $\Omega<4$ suggest that when a topography is in the early stages of growth, a simple multi-Hölder stochastic process may be able to replicate most geomorphically relevant measures of landscape structure. However, when changes in flux become negligible, it is the subtle re-working of a landscape by more diffusive processes that results in an increase in landscape complexity as measured by $\eta^{*}$. This was particularly associated with the coupling between low or intermediate elevations and large Hölder exponents (smooth regions).

The slope-area scaling was also applied to regions of the same area from Florida and Washington State with an order of magnitude difference in elevation range. Despite very different values for $d S / d A$, the values for the GMR control parameter at which there was no significant difference between data and surrogates was very similar $\left(\eta^{*}=0.999\right)$ and very different to the value of $\eta^{*}=0$ expected if a simple multi-Hölder or multifractal model is sufficient to describe the topography. Furthermore, in both cases, as $\eta$ increased from 0.2 to 0.5 , which was the largest change in $d S / d A$ for the Florida data, there was a strong positive correlation between the elevations in the original DEM and the difference in the elevations from the DEMs reconstructed from the fixed wavelet coefficients at $\eta=0.2$ and $\eta=0.5$. These results showed that the primary limitation in the multi-Hölder model was that it was removing too much elevation from the higher altitudes and placing too much at the lower elevations, which 
affected the ability to mimic the observed slope-area scalings.

Our observations raise the question of which class of stochastic processes provides a potential guide to modeling mature landscape surfaces effectively. Our results in Figs. 12 and 13 are explicitly about the coupling between the Hölder exponents and the elevations themselves and that they demonstrate an association implies that self-regulating multi-Hölder surfaces (Lévy Véhel, 2013; Echelard et al., 2015) may have some potential. It also lends support to the recent suggestion that hypsometric analysis can be usefully extended by simultaneous consideration of elevation and Hölder regularity (Keylock et al., 2020b). However, our results also reveal no simple relation between elevation and Hölder regularity, implying further conditioning is necessary. As noted in the introduction, Veneziano and Iacobellis (1999) proposed that differing Hölder regularity could be associated with the channel network and the hillslope and, similar arguments have also been made by Tucker and Bras (1999) and Perron et al. (2008). These ideas are supported by the surrogate data methodology used in this paper. However, such an approach takes us full circle as the introduction began by contrasting geomorphic studies that focus on extracted landscape features with those that attempt to characterize the landscape as a whole, with the latter philosophy guiding the work presented here. Advances in digital terrain processing, (e.g. Passalacqua et al., 2010), simplify the process of DEM classification and the next stage of our work is to form a set of landscape regimes and determine the Hölder conditioning for each, potentially also as a function of elevation. This will lead to a means to determine a statistical modeling framework for natural terrains. The hypothesis testing framework introduced here, or one similar in nature, will be needed to examine the functional relations between landscape regimes and Hölder regularity and, thus, the statistical significance of particular landscape regimes for such a model.

\section{A The classical approach to surrogate data gen- eration}

The philosophy of hypothesis testing for non-linear phenomena using surrogate data was formulated in the 1990s (Theiler et al., 1992; Theiler and Rapp, 1996). In these studies, surrogate data were generated that fulfilled the null hypothesis of being a possible realization of a linear (autocorrelative) process by preserving the Fourier amplitudes and the values of the original signal, but randomizing the Fourier phases. This is known as the iterated, amplitude adjusted Fourier transform (IAAFT) method (Schreiber and Schmitz, 1996). As the surrogates are linearized variants of the original data, comparison of the original data to the surrogates using a suitable metric allows various forms of non-linearity to be

detected. For example, one can determine if the variation in Hölder exponents is sufficient for a signal to be significantly different to monofractal and thereby exhibits statistical intermittency (Poggi et al., 2004; Venema et al., 2006; Basu et al., 2007; Keylock, 2009). 
Given the rejection of such a hypothesis of linearity, gradual reconstruction (Keylock, 2010) can then be used to determine how complex a signal is. In geomorphology, Keylock et al. (2014b) used gradual reconstruction to show how the complexity of river bed topography was a function of discharge, with the superposition of intermediate scale bedforms driving this complexity. Schwenk and Foufoula-Georgiou (2017) used gradual reconstruction to show that the planform of river meanders encodes information on process nonlinearities, with the behavior of pre-cutoff and post-cutoff meander bends contrasted. Beyond geomorphology, Keylock et al. (2015) applied this approach to a multi-Hölder model for turbulence and were able to show the statistical significance of relatively small coefficients in a Fokker-Planck model for the velocity increments.

\section{A.1 An example application of the IAAFT method to our DEMs}
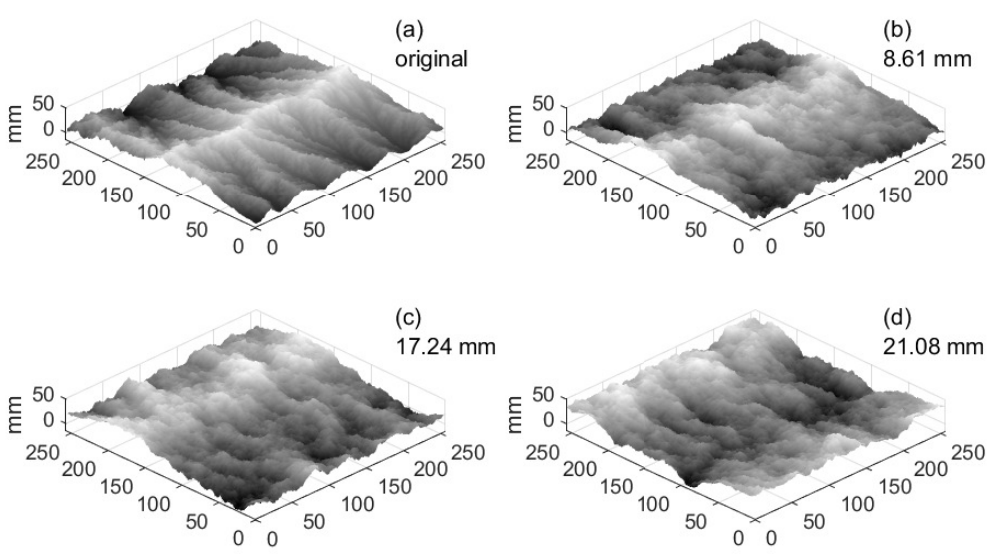

Figure 17: The original DEM at $t=100$ is shown in (a), while (b), (c) and (d) show three surrogate DEMs for this surface generated with the IAAFT algorithm with the minimum, median and maximum root-mean-squared differences in the elevations, $z$, of 19 surrogates.

Figure 17 shows one DEM from our experiment (Singh et al., 2015), together with three example surrogates generated by the Fourier amplitude-preserving IAAFT algorithm. The chosen three DEMs are those with the minimum, median and maximum root-mean-squared differences in elevation between the surrogate DEM and the original DEM at $t=100$. Hence, this figure may be directly compared to Fig. 3, which was based on the same source DEM and had minimum, median and maximum root-mean-squared elevation differences of $5.72 \mathrm{~mm}, 9.91 \mathrm{~mm}$, and $21.43 \mathrm{~mm}$, respectively. The strong visual constrast between the actual terrain and the surrogates implies straightaway that a mono- 
fractal description is inappropriate for describing this surface as a monofractal is statistically equivalent to prescribing a power-law relation for the decay in Fourier amplitudes with frequency. The surrogate data generated in Fig. 3 appear to be much more realistic.
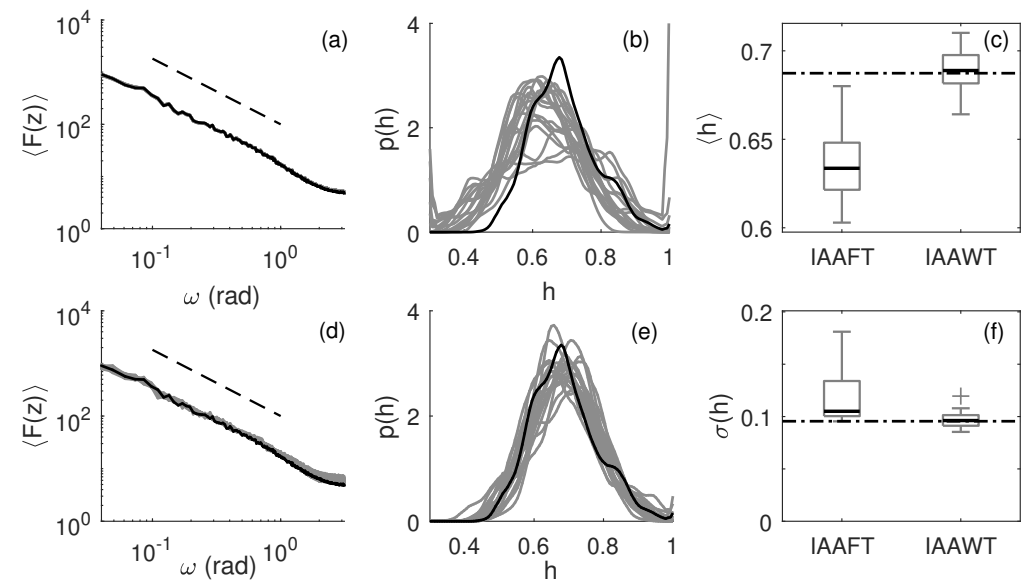

Figure 18: Statistical properties of the original DEM at $t=100$ and the surrogate data. Panels (a) and (b) show results using the IAAFT algorithm and panels (d) and (e) are for the IAAWT algorithm. In each of these four panels, results for the original data are shown in black and for the surrogates in gray. Panels (a) and (d) illustrate the spectral properties of the DEMs with a best-fit power-law shown as a dashed line (displaced vertically). Panels (b) and (e) are the histograms of the pointwise Hölder exponents, $h$. Panels (c) and (f) show boxplots of the values of the mean and standard deviation for $h$, respectively, for nineteen surrogates DEMs generated with the IAAFT and IAAWT algorithms. The values from the original DEM are shown by a horizontal dot-dashed line. The box delimits the lower and upper quartiles with the central bar the median. Whiskers extend for up to 1.5 times the inter-quartile deviation, with outliers shown as crosses.

\section{B Acknowledgements}

This work was supported by Royal Academy of Engineering/Leverhulme Trust Senior Research Fellowship LTSRF1516-12-89 awarded to the lead author, who was also supported by Loughborough University's HPC Hydra cluster. EFG was supported by NSF (grants EAR-1811909 and ECCS-1839441). Digital elevations for the experimental data and the sub-regions of the Ochlockonee River basin and Cowlitz River basin used in this study may be obtained from http://doi.org/10.5281/zenodo.3922330. Code to undertake gradual multifractal reconstruction in two dimensions may be downloaded from 
https://github.com/ChrisKeylock/GradualMultifractalReconstruction.

\section{References}

Ali, N., A. Fuchs, I. Neunaber, J. Peinke, and R. B. Cal (2019), Multiscale/fractal processes in the wake of a wind turbine array boundary layer, Journal of Turbulence, 20(2), 93-120.

Arrell, K. E., P. F. Fisher, N. J. Tate, and L. Bastin, (2007), A fuzzy c-means classification of elevation derivatives to extract the morphometric classification of landforms in Snowdonia, Wales, Comp. Geosci., 33(10), 1366-1381.

Basu, S., E. Foufoula-Georgiou, B. Lashermes, and A. Arneodo (2007), Estimating intermittency exponent in neutrally stratified atmospheric surface layer flows: a robust framework based on magnitude cumulant and surrogate analyses, Phys. Fluids, 19(11), 115102, doi:10.1063/1.2786001.

Benzi, R., L. Biferale, A. Crisanti, G. Paladin, M. Vergassola and A. Vulpiani (1993), A random process for the construction of multiaffine fields, Physica $D, 65,352-358$.

Bonnet, S., and A. Crave (2006), Macroscale dynamics of experimental landscape, in Analogue and Numerical Modelling of Crustal-Scale Processes, Geol. Soc. Spec. Publ., edited by S. J. H. Buiter and G. Schreurs, pp. 327-339, Geol. Soc., London, U. K.

Boon III, J. D. and R. J. Byrne (1981), On basin hypsometry and the morphodynamic response of coastal inlet systems, Marine Geol., 40, 27-48.

Brocklehurst, S. H. and K. X. Whipple (2004), Hypsometry of glaciated landscapes, Earth Surf. Proc. Land., 29, 907-926, doi:10.1002/esp.1083.

Clubb, F. J., S. M. Mudd, D. T. Milodowski, M. D. Hurst, and L. J. Slater (2014), Objective extraction of channel heads from high-resolution topographic data, Water Resour. Res., 50, 4283-4304, doi:10.1002/2013WR015167.

Clubb, F. J., Mudd, S. M., Hurst, M. D., and Grieve, S. W. (2020). Differences in channel and hillslope geometry record a migrating uplift wave at the Mendocino triple junction, California, USA. Geology, 48(2), 184-188.

Cunningham, M.T., C.P. Stark, M.R. Kaplan and J.M. Schaefer, (2019), Glacial limitation of tropical mountain height, Earth Surf. Dynam., 7, 147-169, doi: 10.5194/esurf-7-147-618 2019.

Dietrich, W. E., and J. T. Perron (2006), The search for a topographic signature of life, Nature, 439, 411-418, doi:10.1038/nature04452. 
Echelard, A., J. Lévy Véhel, and A. Philippe (2015), Statistical estimation for a class of self-regulating processes, Scand. J. Stat., 42(2), 485-503.

Ehsani, A. H. and F. Quiel (2008), Geomorphometric feature analysis using morphometric parameterization and artificial neural networks, Geomorphology, 99(1-4), 1-12.

Evans, I. S. and C. J. McLean (1995), The land surface is not unifractal: variograms, cirque scale and allometry, Zeit. für Geomorph. Suppl., 101, 127-147.

Frisch, U. and G. Parisi (1985), The singularity structure of fully developed turbulence, in Turbulence and Predictability in Geophysical Fluid Dynamics and Climate Dynamics edited by M. Ghil, R. Benzi and G. Parisi, pp.84-88, Elsevier.

Gagnon, J.-S., S. Lovejoy, and D. Schertzer (2006), Multifractal earth topography, Nonlin. Proc. Geophys., 13(5), 541-570.

Gailleton, B., Mudd, S. M., Clubb, F. J., Peifer, D., and Hurst, M. D. (2019). A segmentation approach for the reproducible extraction and quantification of knickpoints from river long profiles. Earth Surf. Dyn., 7(1), 211-230.

Gasparini, N.M., and K. X. Whipple (2014), Tectonic control of topography, rainfall patterns, and erosion during rapid post-12 Ma uplift of the Bolivian Andes, Lithosphere 6(4) 251-268, doi:10.1130/L322.1.

Hack, J. T. (1957), Studies of longitudinal stream profiles in Virginia and Maryland, U.S. Geol. Surv. Prof. Pap., 294-B, 1-97.

Hasbargen, L., and C. Paola (2003), How predictable is local erosion rate in erosional landscapes? in Prediction in Geomorphology, Geophys. Monogr. Ser., vol. 136, edited by P. R. Wilcock, and R. M. Iverson, pp. 231-240, AGU, Washington, D. C.

Hooshyar, M., Singh, A., and Wang, D. (2017). Hydrologic controls on junction angle of river networks, Water Resour. Res., 53(5), 4073-4083.

Jaffard, S. (1997), Multifractal formalism for functions .1. Results valid for all functions, SIAM J. Math. Anal., 28, 944-970.

Jerolmack, D.J. and C. Paola (2010), Shredding of environmental signals by sediment transport, Geophys. Res. Lett.37(19), L19401.

Keylock, C. J. (2009), Evaluating the dimensionality and significance of active periods in turbulent environmental flows defined using Lipshitz/Hölder regularity, Environ. Fluid Mech., 9, 509-523.

Keylock, C. J. (2010), Characterizing the structure of nonlinear systems using gradual wavelet reconstruction, Nonlinear Proc.Geophys., 17, 615-632. 
Keylock, C. J. (2012), A resampling method for generating synthetic hydrological time series with preservation of cross-correlative structure and higher order properties, Water Resour. Res., 48(W12521), doi:10.1029/2012WR011923.

Keylock, C. J. (2017), Multifractal surrogate-data generation algorithm that preserves pointwise Holder regularity structure, with initial applications to turbulence, Phys. Rev. E, 95, 032,123.

Keylock, C. J. (2018), Gradual multifractal reconstruction of time-series: Formulation of the method and an application to the coupling between stock market indices and their Hölder exponents, Physica D, 368, 1-9.

Keylock, C. J. (2019), Hypothesis testing for nonlinear phenomena in the geosciences using synthetic, surrogate data, Earth Space Sci., 6, doi: $10.1029 / 2018 \mathrm{EA} 000435$.

Keylock, C. J., M. Ghisalberti, G. G. Katul, and H. M. Nepf (2020a), A joint velocity-intermittency analysis reveals similarity in the vertical structure of atmospheric and hydrospheric canopy turbulence, Env. Fluid Mech., 20, 77101, doi:10.1007/s10652-019-09694-w.

Keylock, C. J., K. Nishimura and J. Peinke (2012), A classification scheme for turbulence based on the velocity-intermittency structure with an application to near-wall flow and with implications for bedload transport, J. Geophys. Res., 117, F01037, doi:10.1029/2011JF002127.

Keylock, C. J., A. Singh, and E. Foufoula-Georgiou (2014b), The complexity of gravel-bed river topography examined with gradual wavelet reconstruction, J. Geophys. Res., 119, 682-700, doi:10.1002/2013JF002999.

Keylock, C. J., A. Singh, P. Passalacqua and E. Foufoula-Georgiou (2020b), Hölder-conditioned hypsometry: A refinement to a classical approach for the characterization of topography, Water Resour. Res

Keylock, C. J., R. Stresing, and J. Peinke (2015) Gradual wavelet reconstruction of the velocity increments for turbulent wakes, Phys. Fluids, 27, art. no. 025104 .

Kingsbury, N. (2001), Complex wavelets for shift invariant analysis and filtering of signals, Appl. Comput. Harmon. Anal., 10, 234-253.

Kirby, E., and Whipple, K. X. (2012). Expression of active tectonics in erosional landscapes. J. Struct. Geol., 44, 54-75.

Klinkenberg, B., and M. F. Goodchild (1992), The fractal properties of topography: A comparison of methods, Earth Surf. Proc. Land., 17(3), 217-234.

Kolmogorov, A. N. (1941), The local structure of turbulence in incompressible viscous fluid for very large Reynolds numbers, Dokl. Akad. Nauk. SSSR., 30, 299-303. 
Lague, D., A. Crave, and P. Davy (2003), Laboratory experiments simulating the geomorphic response to tectonic uplift, J. Geophys. Res., 108, ETG 31-ETG 3-20.

Lague, D. and P. Davy (2003), Constraints on the long-term colluvial erosion law by analyzing slope-area relationships at various uplift rates in the Siwaliks Hills (Nepal), J. Geophys. Res., 108(2), ETG 8-1 - ETG 18-11.

Leuven, J. R. F. W., S. Selaković, and M. G. Kleinhans (2018), Morphology of bar-built estuaries: empirical relation between planform shape and depth distribution, Earth Surf. Dynam., 6, 763-778, https://doi.org/10.5194/esurf6-763-2018.

Lévy Véhel, J. (2013), Beyond multifractional Brownian motion: New stochastic models for geophysical modelling, Nonlin. Proc. Geophys., 20(5), 643-655.

Lifton, N. A., and C. G. Chase (1992), Tectonic, climatic and lithologic influences on landscape fractal dimension and hypsometry: implications for landscape evolution in the San Gabriel Mountains, California, Geomorphology, 5(1-2), 77-114.

Montgomery, D. R. (2001), Slope distributions, threshold hillslopes, and steadystate topography, Am. J. Sci., 301, 432-454.

Montgomery, D. R., and E. Foufoula-Georgiou (1993), Channel network source representation using digital elevation models, Water Resour. Res., 29(12), 3925-3934, doi:10.1029/93WR02463.

Outcalt, S.I. and M. A. Melton (1992), Geomorphic application of the HausdorffBesicovich dimension, Earth Surface Processes and Landforms 17(8), 775-787.

Passalacqua, P., T. Do Trung, E. Foufoula-Georgiou, G. Sapiro, and W. E. Dietrich (2010), A geometric framework for channel network extraction from lidar: Nonlinear diffusion and geodesic paths, J. Geophys. Res, 115 (1), F01002.

Peltier, R. F. and J. Lévy Véhel (1995), Multifractional Brownian motion: definition and preliminary results, INRIA Tech. Report 2645.

Perron, J. T., J. W. Kirchner, and W. E. Dietrich (2008), Spectral signatures of characteristic spatial scales and nonfractal structure in landscapes, J. Geophys. Res., 113(F04003), doi:10.1029/2007JF000866.

Poggi, D., A. Porporato, L. Ridolfi, J. D. Albertson, and G. G. Katul (2004), Interaction between large and small scales in the canopy sublayer, Geophys. Res. Lett., 32(L05102), 10.1029/2003GL018611.

Rodriguez-Iturbe, I., and A. Rinaldo (1997), Fractal River Basins: Chance and Self-Organization, Cambridge Univ. Press, New York.

Schreiber, T., and A. Schmitz (1996), Improved surrogate data for nonlinearity tests, Phys. Rev. Lett., 77, 635-638. 
Schwanghart, W., and Kuhn, N. J. (2010). TopoToolbox: A set of Matlab functions for topographic analysis. Env. Modelling and Software, 25(6), 770781.

Schwenk, J., and E. Foufoula-Georgiou (2017), Are process nonlinearities encoded in meandering river planform morphology?, J. Geophys. Res. Earth Surf., 122, doi:10.1002/2016JF003,929.

Selesnick, I. (2002), The design of approximate Hilbert transform pairs of wavelet bases, IEEE Trans. Sig. Proc., 50, 1144-1152.

Selesnick, I., R. Baraniuk, and N. Kingsbury (2005), The dual-tree complex wavelet transform, IEEE Signal Proc. Mag., 22, 123.

Singh, A., L. Reinhardt, and E. Foufoula-Georgiou (2015), Landscape reorganization under changing climatic forcing: Results from an experimental landscape, Water Resour. Res., 51, 4320-4337, doi:10.1002/2015WR017161.

Strahler, A. N. (1952), Hypsometric (area-altitude) analysis of erosional topography, Geol. Soc. Am. Bull., 63, 1117-1142.

Tarboton, D. G. (1997), A new method for the determination of flow directions and upslope areas in grid digital elevation models, Water Resour. Res., 33(2), 309-319, doi:10.1029/96WR03137.

Tejedor, A., A. Singh, I. Zaliapin, A. L.Densmore, and E. Foufoula-Georgiou (2017), Scale-dependent erosional patterns in steady-state and transient-state landscapes, Sci. Adv., 3, e1701683.

Theiler, J., and P. E. Rapp (1996), Re-examination of the evidence for lowdimensional, nonlinear structure in the human electroencephalogram, Electroencephal. Clin. Neurophys., 98(3), 213-222.

Theiler, J., S. Eubank, A. Longtin, B. Galdrikian, and J. Farmer (1992), Testing for nonlinearity in time series: the method of surrogate data, Physica D, 58, 77-94.

Tokunaga, E. (1978), Consideration on the composition of drainage networks and their evolution, Tokyo Metro. Univ., Japan, Geograph. Rep., 13, 1-27.

Tucker, G. E., and Bras, R. L. (1998). Hillslope processes, drainage density, and landscape morphology. Water Resour. Res., 34 (10), 2751-2764.

Venema, V., S. Meyer, S. G. Garcia, A. Kniffka, C. Simmer, S. Crewell et al. (2006), Surrogate cloud fields generated with the iterative amplitude adapted Fourier transform algorithm, Tellus A, 58, 104-120.

Veneziano, D. and V. Iacobellis (1999), Self-similarity and multifractality of topographic surfaces at basin and subbasin scales, J. Geophys. Res. 104(B6), 12797-12812. 
Venugopal, V., S. Roux, E. Foufoula-Georgiou, and A. Arneodo (2006), Revisiting multifractality of high-resolution temporal rainfall using a wavelet-based formalism, Water Resour. Res. 42(W06D14), doi:10.1029/2005WR004489.

Willgoose, G. (1994), A statistic for testing the elevation characteristics of landscape simulation models, J. Geophys. Res., 99(B7), 13987-13996, doi:10.1029/94JB00123.

Zanardo, S., I. Zaliapin, and E. Foufoula-Georgiou (2013), Are American rivers Tokunaga self-similar? New results on fluvial network topology and its climatic dependence, J. Geophys. Res.: Earth Surf., 118, 1-18, doi:10.1002/jgrf.20029. 